\title{
APLICAÇÃo DO MÉTODO DE ANÁLISE HIERÁRQUICA PARA SELEÇÃo DE SISTEMAS DE SEPARAÇÃO ÁGUA/ÓLEO EM AEROPORTOS PARA DIFERENTES CENÁRIOS
}

\author{
Roberto Gonçalves de Carvalho \\ Divisão de Engenharia Civil - Instituto Tecnológico de Aeronáutica \\ carvargc@yahoo.com.br \\ Nadiane Smaha Kruk \\ Divisão de Engenharia Civil - Instituto Tecnológico de Aeronáutica \\ nadiane@ita.br \\ Mischel Carmen Neyra Belderrain \\ Divisão de Engenharia Mecânica - Instituto Tecnológico de Aeronáutica \\ carmen@ita.br
}

\begin{abstract}
Resumo
A carência de dispositivos normativos e métodos de dimensionamento, aliado à grande parcela de julgamento em engenharia necessária aos tomadores de decisão têm acarretado uma diversidade de soluções técnicas para implantação de Sistemas de Separação Água/Óleo em aeroportos. A grande variabilidade espacial do potencial de ocorrência de vazamentos de óleos e combustíveis constitui-se no principal fator de decisão da solução a adotar, tendo como principais variáveis os critérios custo, espaço ocupado e atendimento da legislação ambiental. Neste trabalho, foi aplicado o método AHP (Método de Análise Hierárquica) com o objetivo de auxiliar administradores de aeroportos na escolha da alternativa mais adequada para implantação desses sistemas, em função do potencial de contaminação que determinada área está sujeita. Foram estabelecidos três cenários típicos, com potenciais de contaminação definidos como baixo, médio e elevado, característicos de áreas com movimentação de aeronaves e veículos de apoio ao solo classificadas como baixa, média e intensa, respectivamente. Os resultados dos julgamentos realizados apontaram para a seleção de sistemas que utilizam separadores API para os cenários com baixo e médio potencial de contaminação e separadores TPI com Tanque de Equalização para o cenário em que há intensa movimentação de aeronaves e veículos.
\end{abstract}

Palavras-Chave: Sistema de Separação Água/Óleo, Método de Análise Hierárquica, aeroportos.

Abstract

The lack of regulatory requirements and design methods, combined with the large amount of engineering judgment needed for decision makers have led to a diversity of technical solutions for implementation of Oil/Water Separation Systems at airports. The large spatial variability of potential spillage of oils and fuels constitutes the main decision factor in the solution to adopt, with the main variables the criteria cost, required space and environmental laws compliance. In this paper, the AHP method (Analytic Hierarchy Process) was applied aiming at assisting airports administrators to choose the most appropriate alternative for deployment, in a function of the potential contamination that particular area is subjected to. Three typical scenarios were established, with potential contamination defined as low, medium and high, characteristics of areas with low, medium and intense aircraft and ground support vehicles movement, respectively. The results of the conducted judgments pointed to choose systems using API separators for scenarios with low and medium contamination potential and TPI separators with equalization tank for the scenario which there is intense movement of aircraft and vehicles.

Keywords: $\quad$ Oil/Water Separation System, Analytic Hierarchy Process, airports. 


\section{INTRODUÇÃO}

A carência de dispositivos normativos e métodos de dimensionamento para projeto de Separadores de Água/Óleo (SAOs), além de proporcionar uma grande dificuldade para os engenheiros projetistas, tem acarretado uma diversidade de soluções técnicas visando a implantação de Sistemas de Separação Água/Óleo (SSAOs) em aeroportos brasileiros.

Conforme estudos de [4], os SAOs instalados para tratamento de efluentes oriundos de lavagem de aeronaves, pisos de hangares, oficinas e postos de abastecimento são facilmente encontrados no mercado e possuem capacidades da ordem de algumas unidades de litros por segundo, uma vez que são fabricados para pequenas vazões de projeto. Por outro lado, os SAOs necessários ao tratamento de efluentes provenientes de águas pluviais que escoam em pátios e pistas de aeroportos, além de apresentarem maiores custos e dificilmente serem encontrados no mercado, apresentam maior porte e ocupam grandes espaços, que muitas vezes são de difícil disponibilidade em áreas aeroportuárias. As vazões dos sistemas de captação das águas pluviais para esses casos são da ordem de centenas de litros por segundo, devendo ser adotados critérios de dimensionamento específicos, de forma a viabilizar a sua implantação. Devido às elevadas vazões resultantes de precipitações em pavimentos aeroportuários, recomenda-se o estudo de alternativas que utilizem o parâmetro first flush (primeira parcela do escoamento superficial) nos dimensionamentos, para estimativa dos volumes de efluentes que deverão ser tratados [4].

O propósito deste trabalho consistiu em utilizar a metodologia multicritério AHP (Analytic Hierarchy Process) para auxiliar os administradores de aeroportos na escolha do SSAO, mais adequado a se implantar em áreas de pistas e pátios de aeroportos, de forma a atender à legislação ambiental, que no Brasil é regulada pela resolução $\mathrm{N}^{\mathbf{o}} 357$ do Conselho Nacional do Meio Ambiente (CONAMA), complementada e alterada pela resolução $\mathrm{N}^{\circ} 430$ do CONAMA, que estabelece a concentração máxima para lançamento de efluentes contaminados com óleos minerais provenientes de qualquer fonte poluidora em $20 \mathrm{mg} / \mathrm{L}$.

\section{REVISÃO BIBLIOGRÁFICA}

\subsection{Sistemas de SeParaÇão ÁGUa/Óleo}

Segundo [5], os SSAO em aeroportos têm como objetivo a coleta de um efluente contaminado com graxa, óleos lubrificantes e combustíveis de aeronaves, veículos e equipamentos de apoio ao solo através de um sistema de captação dotado de calhas, canaletas, valas, tubulações, e outros dispositivos de drenagem, e seu posterior tratamento pela passagem desse efluente através de um SAO, que realiza por diferenças de massa específica entre os materiais, a separação da matéria contaminante da água, até um nível de concentração definido em projeto, que possibilite o seu lançamento ao meio ambiente.

Conforme estudos de [2], um dos aspectos considerados na escolha de um SAO em aeroportos está relacionado à manutenção e à especialização de pessoal para operações de retro lavagem. Levando em consideração os custos de operação, os SAOs mais empregados e existentes no mercado restringem-se a dois tipos: os separadores tipo API, que seguem a Publicação $\mathrm{n}^{\circ} 421$ do American Petroleum Institute [1] e os separadores com placas coalescentes, também chamados de TPI (Tilted Plate Interceptor).

Os separadores API são formados por três câmaras, sendo a primeira para sedimentação de partículas sólidas presentes no fluxo, a segunda para deposição do óleo na superfície da lâmina líquida e a terceira para descarga. Os separadores com placas coalescentes ou TPI são formados pela superposição de inúmeras placas paralelas onduladas paralelas, inclinadas de $45^{\circ}$ a $60^{\circ}$ e separadas entre si de 20 a $40 \mathrm{~mm}$. Essa superposição produz um aumento de área efetiva de separação entre o óleo e a água, resultando em unidades compactas de menor volume. 
Para dimensionamento dos SSAO, primeiramente deve-se definir se a caixa separadora será posicionada em linha (on line) ou fora de linha (off line). Sistemas em linha são projetados para atender a vazões de pico das áreas de contribuição. Objetivando reduzir o tamanho das estruturas dos separadores em linha e garantir que a parcela inicial do escoamento superficial (first flush) que contém a maior concentração de óleos e combustíveis fosse tratada, [5] propôs uma metodologia para dimensionamento de SSAO que utiliza separadores TPI com Tanques de Equalização (TE). Os resultados mostraram uma significativa redução de área e custo, bem como um aumento na eficiência de remoção, em relação a sistemas que utilizam separadores TPI em linha, ou outros tipos de separadores, como o API ou o TPI, posicionados fora de linha.

\subsection{MÉTOdO DE ANÁLISE HIERÁRQUICA - AHP}

Conforme [13], os primeiros métodos de Apoio Multicritério à Tomada de Decisão (MCDA) surgiram na década de 70. Esses métodos podem ser divididos em dois ramos: o contínuo e o discreto. No ramo contínuo, também chamado Programação Multi-objetivo, as alternativas podem adquirir um número infinito de valores. No ramo discreto, denominado Decisão Multicritério Discreta (DMD), os problemas têm um número finito de alternativas.

O Método de Análise Hierárquica (AHP) foi desenvolvido em 1980 por Thomas L. Saaty para auxiliar na resolução de problemas complexos de tomada de decisão [8]. O AHP basicamente estrutura o problema em níveis hierárquicos, iniciando pelos objetivos seguidos dos critérios, depois sub-critérios e finalmente no último nível as alternativas [11]. Segundo [7] o AHP foi um dos primeiros métodos no ramo das DMDs, e talvez o mais utilizado no mundo. Vaidya e Kumar [12] publicaram um estudo de revisão de literatura das aplicações do método multicritério AHP. Nesse estudo foram revistas mais de 150 aplicações do AHP nos mais variados setores evidenciando que o método pode ser aplicado em quase todos os problemas que envolvem a tomada de decisão.

Segundo [10], para se tomar uma decisão e gerar prioridades de forma organizada o problema de decisão deverá ser decomposto nas seguintes etapas:

1. Definição do problema e determinação do tipo de conhecimento que se busca;

2. Estruturação da uma hierarquia de decisão, desde o topo, para o qual é definido o objetivo da decisão, seguido dos níveis intermediários que constituem os critérios e os subcritérios, até o nível mais baixo, caracterizado pelo conjunto das possíveis alternativas;

3. Construção de um conjunto de matrizes de comparação par a par, onde cada elemento de um nível superior é usado na comparação dos elementos do nível imediatamente inferior à luz do primeiro;

4. Uso das prioridades obtidas através das comparações para atribuir pesos e definir prioridades dos níveis imediatamente abaixo. Em seguida, para cada elemento do nível inferior, soma-se seu peso para obtenção do valor global de prioridade.

Para fazer as comparações, Saaty propõe uma escala de números, da qual foi denominada Escala Fundamental de Saaty (Tabela 1) que indica quantas vezes mais importante ou dominante é um elemento em relação a outro elemento com respeito ao critério ou propriedade em relação à qual eles são comparados. O motivo para se trabalhar com a lógica ilustrada na Tabela 1 é a existência do denominado limite psicológico, segundo o qual o ser humano pode, no máximo, julgar corretamente $7 \pm 2$ pontos, ou seja, nove pontos para distinguir essas diferenças. Saaty acrescenta ainda, que outro motivo da existência desta tabela ser de 1-9 é a habilidade para fazer distinções qualitativas, muito bem representadas entre os cinco atributos: igual, fraco, grande, muito grande e absoluta. Nesta situação, uma pessoa pode fazer a relação entre os atributos com grande precisão [3]. 
Tabela 1 - Escala Fundamental de Saaty.

\begin{tabular}{|c|c|c|}
\hline $\begin{array}{c}\text { Intensidade de } \\
\text { Importância }\end{array}$ & Definição & Explicação \\
\hline 1 & Mesma importância & As duas atividades contribuem igualmente para o objetivo. \\
\hline 3 & $\begin{array}{l}\text { Importância pequena de uma sobre a } \\
\text { outra }\end{array}$ & $\begin{array}{l}\text { A experiência e o julgamento favorecem levemente uma } \\
\text { atividade em relação a outra. }\end{array}$ \\
\hline 5 & Importância grande ou essencial & $\begin{array}{l}\text { A experiência e o julgamento favorecem fortemente uma } \\
\text { atividade em relação a outra. }\end{array}$ \\
\hline 7 & Importância grande ou demonstrada & $\begin{array}{l}\text { Uma atividade é muito fortemente favorecida em relação a } \\
\text { outra; sua denominação de importância é demonstrada na } \\
\text { prática }\end{array}$ \\
\hline 9 & Importância extrema ou absoluta & $\begin{array}{l}\text { Quando se procura uma condição de compromisso entre } \\
\text { duas definições }\end{array}$ \\
\hline $2,4,6,8$ & $\begin{array}{c}\text { Valores intermediários entre os valores } \\
\text { adjacentes }\end{array}$ & Quando se procura uma condição entre duas definições \\
\hline
\end{tabular}

A comparação par a par é feita através de matrizes quadradas de julgamento onde os números na linha i e na coluna $\mathrm{j}$ representam a importância do critério $\mathrm{C}_{\mathrm{i}}$ em relação ao critério $\mathrm{C}_{\mathrm{j}}$, conforme a estrutura matricial representada abaixo:

$$
A=\left[\begin{array}{ccccc}
1 & a_{12} & a_{13} & \ldots & a_{1 i} \\
1 / a_{12} & 1 & a_{23} & \ldots & a_{2 j} \\
1 / a_{13} & 1 / a_{23} & 1 & \ldots & a_{3 j} \\
\ldots & \ldots & \ldots & 1 & \ldots \\
1 / a_{1 j} & 1 / a_{2 j} & 1 / a_{3 j} & \ldots & 1
\end{array}\right]
$$

Após a obtenção das matrizes de julgamento procede-se à normalização destas matrizes, cujos elementos são obtidos por meio da divisão de cada elemento da matriz de julgamento pelo somatório dos elementos da coluna a qual pertence o respectivo elemento. A matriz resultante deste processo é denominada Matriz Normalizada (MN) e é definida por:

$$
A^{\prime}=\left[a_{i j}^{\prime}\right] \text {, tal que } a_{i j}^{\prime}=a_{i j} / \sum_{k=1}^{n} a_{i k} \text { para } 1 \leq i \leq n, e 1 \leq j \leq n
$$

Em seguida, procede-se ao cálculo da Prioridade Média Local (PML), onde as MNs são manipuladas para obtenção das prioridades relativas de cada um dos critérios. A PML é obtida a partir do cálculo das médias das linhas da $\mathrm{MN}$, sendo denominada de vetor de prioridade local (autovetor). A PML é a matriz determinada por:

$$
W=\left[W_{k}\right] \text {, tal que } w_{k}=\sum_{k=1}^{n} a_{i k}^{\prime} / n \text { para } 1 \leq i \leq n, e 1 \leq j \leq n
$$

Após o cálculo da PML, o próximo passo do método AHP é a obtenção da Prioridade Global (PG). Para calcular a PG é necessário combinar as PMLs relativas das alternativas com os critérios, com o intuito de produzir um vetor de prioridades compostas que servirá como prioridade das alternativas de decisão na busca do objetivo principal do problema. Uma vez que todos os pesos relativos tenham sido calculados na PML, uma PG (ou peso composto " $\mathrm{C}_{\mathrm{d}}$ ") para cada escolha da decisão (chamada "d") é determinada. Isso é definido pela agregação de pesos sobre a hierarquia para cada escolha da decisão. Para tanto, multiplica-se o peso através do caminho, desde o topo da hierarquia, descendo até as escolhas da decisão, e então, soma-se esses produtos de todos os diferentes caminhos da escolha da decisão. $\mathrm{O}$ resultado é um único valor de peso para cada escolha da decisão. Pela notação matemática, o peso composto, $\mathrm{C}$, é dado por:

$$
C=\left[c_{d}\right] \text { para } 1 \leq d \leq n \text {, tal que } c_{d}=\sum_{t=1}^{n t} W_{t} \cdot \prod_{l=1}^{n l-1} W_{l}
$$


Como a base do método AHP é a realização de um julgamento de valor, pode-se esperar que ocorram avaliações inconsistentes em algumas situações. Prevendo esta possibilidade, o método desenvolvido por [8] propõe procedimentos que permitem avaliar a consistência dos julgamentos. O primeiro deles é o Índice de Consistência (IC), que pode ser calculado por meio da Equação (1).

$$
I C=\frac{\left|\lambda_{\max }-N\right|}{N-1}
$$

Em que $N$ é a ordem da matriz e $\lambda_{\max }$ é o maior auto valor da matriz de julgamentos paritários.

O segundo é a Razão de Consistência (RC), que permite avaliar a inconsistência em função da ordem da matriz de julgamentos, e pode ser calculado pela Equação (2).

$$
R C=\frac{I C}{I R}
$$

Em que IC representa o Índice de Consistência e IR é o Índice Randômico.

$\mathrm{O}$ valor de IR para matrizes de diferentes ordens foram aproximados por Saaty para vários tamanhos de matriz $\mathrm{N}$ e são apresentados na Tabela 2.

Tabela 2 - Índices de Consistência Randômicos (IR)

\begin{tabular}{|c|c|c|c|c|c|c|c|c|c|c|c|}
\hline Ordem da Matriz (N) & 1 & 2 & 3 & 4 & 5 & 6 & 7 & 8 & 9 & 10 & 11 \\
\hline Valores de IR & 0 & 0 & 0,58 & 0,9 & 1,12 & 1,24 & 1,32 & 1,41 & 1,45 & 1,49 & 1,51 \\
\hline
\end{tabular}

\section{METODOLOGIA}

\subsection{Estruturação do Problema e Definição dos CenÁRios}

Para estruturar o problema e determinar o tipo de solução a que se busca, foram realizadas reuniões entre profissionais com expertise em infraestrutura de drenagem e saneamento em aeroportos, onde, por meio de brainstorming e escolhas baseadas no consenso, definiram-se os principais componentes de uma estrutura de decisão com o objetivo de selecionar um SSAO a ser implantado em uma determinada área aeroportuária com certo potencial de contaminação por óleo. A escolha de um SSAO envolve o estudo das principais fontes poluentes de óleos, graxas e combustíveis nas diversas áreas de um aeroporto, sujeitas à movimentação de aeronaves e veículos diversos, que podem ser para apoio à manutenção, abastecimento, combate a incêndio e segurança, bem como de equipamentos de rampa, como macacos hidráulicos, escadas, dentre outros. Como a probabilidade de ocorrência de vazamentos de contaminantes oleosos em determinada área varia de acordo com a frequência de movimentação dessas fontes poluidoras, foram definidos três cenários nos quais puderam ser atribuídos diferentes potenciais de ocorrência desses contaminantes.

O Cenário 1 foi definido para situações em que a possibilidade de ocorrência de contaminação por óleos ou hidrocarbonetos é baixa. Para esta situação, as áreas sujeitas à movimentação de aeronaves e veículos que permanecem por pouco tempo sob os pavimentos ou que representam passagens rápidas com altas velocidades (acima de $80 \mathrm{~km} / \mathrm{h}$ ), apresentam potencial baixo para ocorrência de vazamentos de óleos e combustíveis sob os pavimentos, e portanto, estarão sujeitas a parâmetros de projeto mais específicos em função do risco de contaminação ao qual estão expostas. As áreas de pistas de pouso e decolagem são as mais sujeitas a este cenário, uma vez que as aeronaves e veículos que nela transitam operam com altas velocidades, constituindo-se em baixo potencial por contaminação oleosa. O Cenário 2 compreende as situações em que a passagem de aeronaves e veículos de apoio ao solo ocorrem em um intervalo de tempo moderado sob determinada área. Esta situação ocorre, para o caso de movimentação de aeronaves, durante um taxiamento ou quando a mesma aguarda uma posição no pátio para estacionamento, e no caso de veículos, durante a realização de uma inspeção ou ronda. Em geral, esta situação é característica dos locais em 
que as velocidades operacionais variam de 0 a $80 \mathrm{~km} / \mathrm{h}$, incluindo pequenas paradas. As pistas de taxiamento e cabeceiras de pistas de pouso e decolagem são áreas típicas para ocorrência deste cenário. Por fim, o Cenário 3 caracteriza-se pela ocorrência de situações onde as aeronaves e veículos de apoio ao solo permanecem estacionados por extensos intervalos de tempo, ou em que haja tráfego ou movimentação bastante intensa. Neste caso, as áreas de pátio de aeronaves e as áreas de estacionamento de veículos de apoio são os exemplos típicos para caracterização deste cenário.

\subsection{CRITÉrios}

Conforme já mencionado anteriormente, a carência de dispositivos normativos e métodos de dimensionamento para projeto de SAOs têm acarretado uma diversidade de soluções técnicas visando à implantação SSAO em aeroportos. Além disso, a falta de critérios e condicionantes para escolhas dos SSAOs muitas vezes tem levado à adoção de soluções equivocadas que dificilmente atendem aos requisitos estabelecidos na legislação ambiental brasileira. Visando estabelecer um procedimento racional para a implantação de SSAO, e com base na técnica de brainstorming realizada entre profissionais da área de saneamento e drenagem aeroportuária, foram definidos cinco critérios mais representativos para auxílio na escolha do tipo de SSAO a implantar, selecionados com base nas propriedades de exaustividade, não-redundância, coesão, operacionalidade, legitimidade e consistência, sugeridas por [6] para escolha de critérios em problemas de decisão multicritério. São eles:

- Custo - Dada a grande variabilidade nos custos das diversas soluções ou alternativas de implantação de SSAO, este critério foi apontado como o principal limitador para a tomada de decisão;

- Espaço - A implantação de SSAO envolve a definição e reserva de espaço, que muitas vezes são de difícil disponibilidade em áreas aeroportuárias. Desta forma, em função da disponibilidade de área e do espaço necessário para implantação de determinado tipo de SSAO, este critério pode representar diferentes julgamentos para distintos cenários;

- Eficiência - A eficiência de remoção dos contaminantes oleosos provenientes do escoamento superficial sobre áreas pavimentadas de aeroportos está diretamente relacionada ao tipo de SSAO implantado para determinada situação potencial de ocorrência de derramamento de óleo, ou hidrocarbonetos;

- Atendimento à resolução CONAMA 357/430 - A necessidade de atendimento aos requisitos da legislação ambiental para lançamento dos efluentes contaminados com óleos e graxas em áreas aeroportuárias deverá ser avaliado na escolha dos SSAO;

- Manutenção - A chave para o desempenho de um SSAO é manutenção. Quando não mantidos adequadamente por falta de procedimentos de manutenção, os SAOs poderão apresentar problemas de entupimento e ou até mesmo, estruturais. A necessidade de mão de obra especializada e os procedimentos de manutenção são os principais variantes na escolha de implantação de um SSAO.

\subsection{Alternativas}

As alternativas foram selecionadas com base em soluções tradicionalmente empregadas em aeroportos. Além dos modelos existentes, foram propostas alternativas que apresentam soluções e tecnologias que se encontram em desenvolvimento e que apresentam grande potencial para aumento da eficiência, redução de custo e de espaço, bem como atendimento à resolução CONAMA 357/430. Quatro alternativas foram selecionadas com base na técnica de brainstorming realizada entre profissionais de saneamento e drenagem aeroportuária. São elas:

- API - Esta alternativa utiliza como solução um SSAO que emprega um separador do tipo API. Esta tecnologia é da década de 1990, porém ainda é utilizada por apresentar 
baixos custos em relação aos demais tipos de separadores empregados, tanto de implantação quanto de manutenção. As desvantagens estão relacionadas à baixa eficiência e grande necessidade de espaço;

- TPI - Os SSAO que utilizam separadores TPI como alternativa são mais eficientes e ocupam menores áreas que os API, porém apresentam maiores custos.

- TPI em linha - São utilizados em SSAO dimensionados para tratamento da vazão de pico. São bastante eficientes, porém ocupam grandes espaços e apresentam custos elevados em relação aos demais tipos;

- TPI com TE - Esta alternativa de SSAO emprega separadores TPI associados a TE. Apresentam mesmo nível de eficiência que os sistemas que empregam o TPI em linha. O TE, tem como objetivo, uma redução na área do sistema, resultando menores custos em relação ao TPI em linha.

\subsection{ESTRUTURAÇÃO dOS NÍVEIS HIERÁRQUICOS}

Após as etapas de definição dos critérios e alternativas, foi montada a estruturação em nível hierárquico, onde é possível visualizar os critérios para escolha do SSAO para cada cenário em análise, conforme apresentado na Figura 1.

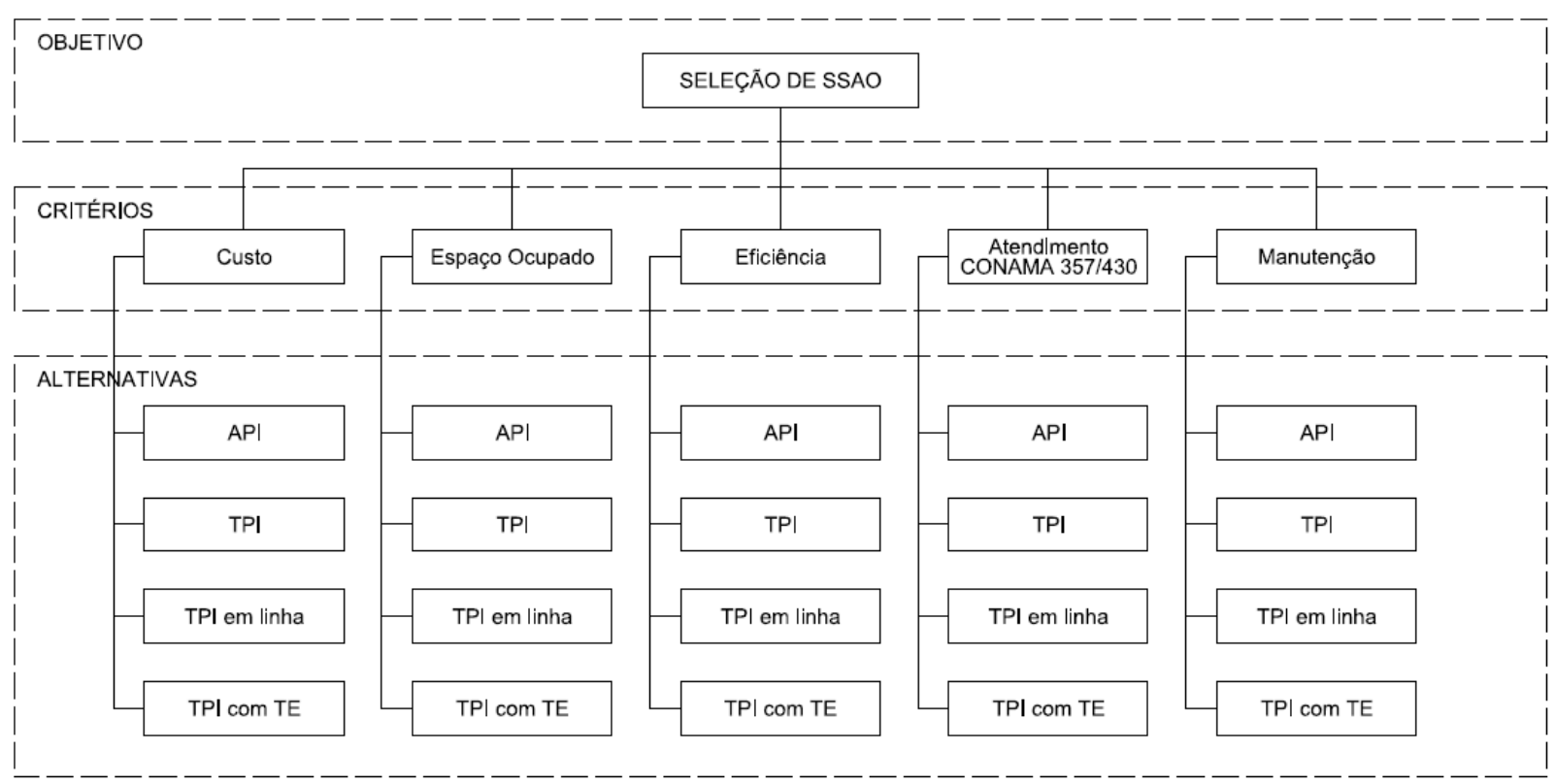

Figura 1 - Estrutura hierárquica para a seleção de SSAO

Fonte: Elaborado pelos autores.

\section{RESULTADOS E DISCUSSÕES}

Para avaliar os critérios em relação ao objeto, e as alternativas em relação aos critérios para cada cenário, foi utilizada a Escala Fundamental de Saaty, conforme valores apresentados na Tabela 1. Os julgamentos foram realizados por meio do consenso entres os profissionais das áreas de saneamento e drenagem aeroportuária participantes do processo. Em seguida foi avaliada a consistência dos julgamentos e promovidas pequenas revisões nas matrizes de comparação de forma a obter Razões de Consistências inferiores a 0,1. A seguir são apresentados os resultados para os três cenários avaliados.

\subsection{Cenário 1}

As matrizes de julgamento das alternativas propostas em relação a cada critério, bem como os valores da Razão de Consistência dos julgamentos para a situação característica do Cenário 1 são apresentados no Quadro 1. 
Quadro 1 - Matrizes de julgamento das alternativas em relação aos critérios, para o Cenário 1.

\begin{tabular}{|c|c|c|c|c|}
\hline CUSTO & $\bar{z}$ & $\overline{\mathrm{G}}$ & 愛 & 产 \\
\hline API & 1 & 5 & 7 & 5 \\
\hline TPI & $1 / 5$ & 1 & 3 & 3 \\
\hline TPI em linha & $1 / 7$ & $1 / 3$ & 1 & $1 / 3$ \\
\hline TPI com TE & $1 / 5$ & $1 / 3$ & 3 & 1 \\
\hline \multicolumn{5}{|c|}{$\lambda_{\max }=4,235 ; \mathrm{IC}=0,078 ; \mathrm{RC}=0,087$} \\
\hline
\end{tabular}

\begin{tabular}{|c|c|c|c|c|}
\hline ESPAÇO & $\bar{\xi}$ & $\bar{E}$ & 吾 & 言 \\
\hline API & 1 & $1 / 3$ & 3 & 1 \\
\hline TPI & 3 & 1 & 5 & 1 \\
\hline TPI em linha & $1 / 3$ & $1 / 5$ & 1 & $1 / 5$ \\
\hline TPI com TE & 1 & 1 & 5 & 1 \\
\hline
\end{tabular}

\begin{tabular}{|c|c|c|c|c|}
\hline EFICIÊNCIA & $\bar{z}$ & $\overline{\underline{Z}}$ & 吾 & 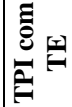 \\
\hline API & 1 & $1 / 2$ & $1 / 3$ & $1 / 4$ \\
\hline TPI & 2 & 1 & $1 / 3$ & $1 / 3$ \\
\hline TPI em linha & 3 & 3 & 1 & 1 \\
\hline TPI com TE & 4 & 3 & 1 & 1 \\
\hline \multicolumn{5}{|c|}{$\lambda_{\max }=4,046 ; \mathrm{IC}=0,015 ; \mathrm{RC}=0,017$} \\
\hline
\end{tabular}

\begin{tabular}{|c|c|c|c|c|}
\hline $\begin{array}{c}\text { ATEND. } \\
\text { CONAMA } \\
\text { 357/430 }\end{array}$ & \& & $\overline{\underline{T}}$ & 吾 & 言 \\
\hline API & 1 & $1 / 3$ & $1 / 5$ & $1 / 4$ \\
\hline TPI & 3 & 1 & $1 / 3$ & $1 / 3$ \\
\hline TPI em linha & 5 & 3 & 1 & 2 \\
\hline TPI com TE & 4 & 3 & 0,5 & 1 \\
\hline \multicolumn{5}{|c|}{$\lambda_{\max }=4,109 ; \mathrm{IC}=0,036 ; \mathrm{RC}=0,040$} \\
\hline
\end{tabular}

\begin{tabular}{|c|c|c|c|c|}
\hline MANUTEN. & ( & & & \\
& & & & \\
\hline API & 1 & 5 & 7 & 5 \\
\hline TPI & $1 / 5$ & 1 & 4 & 1 \\
\hline TPI em linha & $1 / 7$ & $1 / 4$ & 1 & $1 / 3$ \\
\hline TPI com TE & $1 / 5$ & 1 & 3 & 1 \\
\hline$\lambda_{\max }=4,114 ;$ IC $=0,040 ; \mathrm{RC}=0,042$ \\
\hline
\end{tabular}

O Quadro 2 apresenta a matriz de julgamento dos critérios avaliados em relação ao objetivo e a Razão de Consistência resultante deste julgamento, assim como os valores de Prioridade Média Local, Prioridade Global e o resultado final para o Cenário 1.

Quadro 2 - Matriz de julgamento dos critérios em relação ao objetivo, vetores Prioridade Média Local e Prioridade Global e resultado da seleção do SSAO, para o Cenário 1.

\begin{tabular}{|c|c|c|c|c|c|}
\hline $\begin{array}{c}\text { SELEÇÃO } \\
\text { DE SSAOO }\end{array}$ & 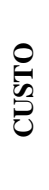 & 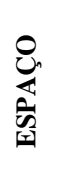 & 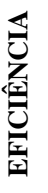 & 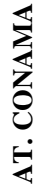 & 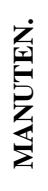 \\
\hline CUSTO & 1 & 8 & 7 & 5 & 7 \\
\hline ESPAÇO & $1 / 8$ & 1 & 5 & 3 & 2 \\
\hline EFICIÊNCIA & $1 / 7$ & $1 / 5$ & 1 & $1 / 3$ & $1 / 2$ \\
\hline AT. CONAMA & $1 / 5$ & $1 / 3$ & 3 & 1 & 1 \\
\hline MANUTENÇÃO & $1 / 7$ & $1 / 2$ & 2 & 1 & 1 \\
\hline$\lambda_{\max }=$ & & & & & \\
\hline
\end{tabular}

\begin{tabular}{|c|c|c|c|c|c|c|}
\hline \multirow[b]{2}{*}{$\begin{array}{c}\text { SELEÇÃO } \\
\text { DE SSAO }\end{array}$} & \multicolumn{5}{|c|}{ VETORES PRIORIDADE - PML } & \multirow[b]{2}{*}{ 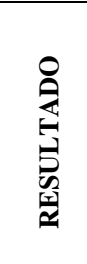 } \\
\hline & $\begin{array}{l}0 \\
0 \\
0 \\
0\end{array}$ & 导 & 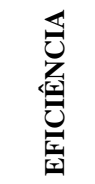 & 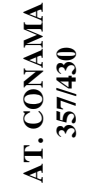 & 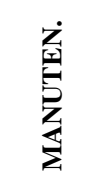 & \\
\hline API & 0,6085 & 0,2115 & 0,0971 & 0,0726 & 0,6216 & 0,4577 \\
\hline TPI & 0,2038 & 0,4067 & 0,1468 & 0,1560 & 0,1676 & $\mathbf{0 , 2 3 0 4}$ \\
\hline TPI em linha & 0,0624 & 0,0688 & 0,3655 & 0,4609 & 0,0598 & 0,1184 \\
\hline TPI com TE & 0,1253 & 0,3130 & 0,3905 & 0,3104 & 0,1510 & 0,1935 \\
\hline $\begin{array}{c}\text { Prioridade } \\
\text { Global - PG }\end{array}$ & 0,5799 & $\mathbf{0 , 1 8 3 9}$ & 0,0480 & 0,1016 & 0,0867 & \\
\hline
\end{tabular}

Conforme os valores da Prioridade Global, observa-se que para a situação característica do Cenário 1, o custo foi o critério que obteve o maior peso, representando $58 \%$ da preferência no julgamento realizado, seguido do critério espaço, que representou cerca de $18 \%$ de prioridade para escolha do SSAO. Neste cenário, o critério eficiência foi o que obteve o menor peso no julgamento, representando menos que 5\% da preferência. Os pesos dos critérios Atendimento da Resolução CONAMA 357/430 e Manutenção representaram cerca de $10 \%$ e $9 \%$, do peso no julgamento, respectivamente.

\subsection{CENÁRIO 2}

As matrizes de julgamento das alternativas propostas em relação a cada critério, bem como os valores da Razão de Consistência dos julgamentos para a situação característica do Cenário 2 são apresentados no Quadro 3. 
Quadro 3 - Matrizes de julgamento das alternativas em relação aos critérios, para o Cenário 2.

\begin{tabular}{|c|c|c|c|c|}
\hline CUSTO & $\bar{z}$ & $\overline{\mathrm{G}}$ & 愛 & 产 \\
\hline API & 1 & 3 & 7 & 5 \\
\hline TPI & $1 / 3$ & 1 & 4 & 3 \\
\hline TPI em linha & $1 / 7$ & $1 / 4$ & 1 & $1 / 4$ \\
\hline TPI com TE & $1 / 5$ & $1 / 3$ & 4 & 1 \\
\hline \multicolumn{5}{|c|}{$\lambda_{\max }=4,140 ; \mathrm{IC}=0,065 ; \mathrm{RC}=0,072$} \\
\hline
\end{tabular}

\begin{tabular}{|c|c|c|c|c|}
\hline ESPAÇO & $\overline{4}$ & $\overline{\underline{G}}$ & 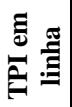 & 递 \\
\hline API & 1 & $1 / 5$ & $1 / 3$ & $1 / 2$ \\
\hline TPI & 5 & 1 & 5 & 3 \\
\hline TPI em linha & 3 & $1 / 5$ & 1 & $1 / 3$ \\
\hline TPI com TE & 2 & $1 / 3$ & 3 & 1 \\
\hline \multicolumn{5}{|c|}{$\lambda_{\max }=4,257 ; \mathrm{IC}=0,086 ; \mathrm{RC}=0,095$} \\
\hline
\end{tabular}

\begin{tabular}{|c|c|c|c|c|}
\hline EFICIÊNCIA & $\bar{Q}$ & $\bar{C}$ & 窇 & 氮 \\
\hline API & 1 & $1 / 5$ & $1 / 7$ & $1 / 6$ \\
\hline TPI & 5 & 1 & $1 / 5$ & $1 / 4$ \\
\hline TPI em linha & 7 & 5 & 1 & 2 \\
\hline TPI com TE & 6 & 4 & $1 / 2$ & 1 \\
\hline
\end{tabular}

\begin{tabular}{|c|c|c|c|c|}
\hline $\begin{array}{c}\text { ATEND. } \\
\text { CONAMA } \\
\text { 357/430 }\end{array}$ & 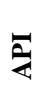 & $\overline{\mathrm{S}}$ & 产 & 焉 \\
\hline API & 1 & $1 / 3$ & $1 / 5$ & $1 / 5$ \\
\hline TPI & 3 & 1 & $1 / 3$ & $1 / 3$ \\
\hline TPI em linha & 5 & 3 & 1 & 1 \\
\hline TPI com TE & 5 & 3 & 1 & 1 \\
\hline \multicolumn{5}{|c|}{$\lambda_{\max }=4,044 ; \mathrm{IC}=0,015 ; \mathrm{RC}=0,016$} \\
\hline
\end{tabular}

\begin{tabular}{|c|c|c|c|c|}
\hline MANUTEN. & $\overline{\mathrm{z}}$ & $\overline{\underline{E}}$ & $\stackrel{g}{\Xi}$ & 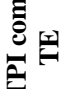 \\
\hline API & 1 & 3 & 7 & 5 \\
\hline TPI & $1 / 3$ & 1 & 5 & 3 \\
\hline TPI em linha & $1 / 7$ & $1 / 5$ & 1 & $1 / 4$ \\
\hline TPI com TE & $1 / 5$ & $1 / 3$ & 4 & 1 \\
\hline \multicolumn{5}{|c|}{$\lambda_{\max }=4,182 ; \mathrm{IC}=0,061 ; \mathrm{RC}=0$} \\
\hline
\end{tabular}

O Quadro 4 apresenta a matriz de julgamento dos critérios avaliados em relação ao objetivo e a Razão de Consistência resultante deste julgamento, assim como os valores de Prioridade Média Local, Prioridade Global e o resultado final para o Cenário 2.

Quadro 4 - Matriz de julgamento dos critérios em relação ao objetivo, vetores Prioridade Média Local e Prioridade Global e resultado da seleção do SSAO, para o Cenário 2.

\begin{tabular}{|c|c|c|c|c|c|}
\hline $\begin{array}{c}\text { SELEÇÃO } \\
\text { DE SSAOO }\end{array}$ & $\begin{array}{l}0 \\
0 \\
0 \\
0\end{array}$ & 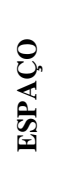 & 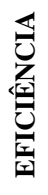 & 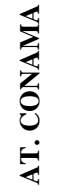 & 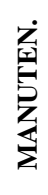 \\
\hline CUSTO & 1 & 3 & 5 & 3 & 2 \\
\hline ESPAÇO & $1 / 3$ & 1 & 3 & 2 & 1 \\
\hline EFICIÊNCIA & $1 / 5$ & $1 / 3$ & 1 & $1 / 2$ & $1 / 3$ \\
\hline AT. CONAMA & $1 / 3$ & $1 / 2$ & 2 & 1 & 2 \\
\hline MANUTENÇÃO & $1 / 2$ & 1 & 3 & $1 / 2$ & 1 \\
\hline
\end{tabular}

\begin{tabular}{|c|c|c|c|c|c|c|}
\hline \multirow[b]{2}{*}{$\begin{array}{c}\text { SELEÇÃO } \\
\text { DE SSAOO }\end{array}$} & \multicolumn{5}{|c|}{ VETORES PRIORIDADE - PML } & \multirow[b]{2}{*}{ 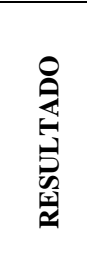 } \\
\hline & $\begin{array}{l}0 \\
\stackrel{0}{0} \\
0\end{array}$ & 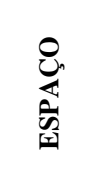 & 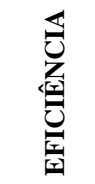 & 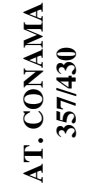 & 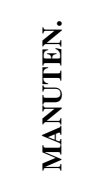 & \\
\hline API & 0,5573 & 0,0864 & 0,0496 & 0,0687 & 0,5527 & $\mathbf{0 , 3 5 0 0}$ \\
\hline TPI & 0,2478 & 0,5470 & 0,1357 & 0,1535 & 0,2595 & 0,2846 \\
\hline TPI em linha & 0,0573 & 0,1411 & 0,4967 & 0,3889 & 0,0538 & $\mathbf{0 , 1 5 7 5}$ \\
\hline TPI com TE & 0,1375 & 0,2256 & 0,3180 & 0,3889 & 0,1341 & $\mathbf{0 , 2 0 7 9}$ \\
\hline $\begin{array}{c}\text { Prioridade } \\
\text { Global - PG }\end{array}$ & 0,4077 & 0,1940 & 0,0674 & 0,1656 & 0,1653 & \\
\hline
\end{tabular}

Da observação dos valores da Prioridade Global para esta a situação, constatou-se que o custo, assim como para o Cenário 1, foi o critério que obteve o maior peso, representando cerca de $41 \%$ da preferência no julgamento realizado, porém, nesse caso, com menos intensidade que no cenário anterior. Com relação ao critério espaço, que representou $19 \%$ da prioridade para escolha do SSAO, não houve muita variação em relação ao primeiro cenário. Os pesos dos critérios Atendimento da Resolução CONAMA 357/430 e Manutenção representaram cada um, 16,5\% do peso no julgamento, evidenciando um aumento na prioridade destes dois critérios em relação ao custo. Assim como no cenário anterior, o critério eficiência foi o que obteve o menor peso no julgamento, representando menos que $7 \%$ da preferência.

\subsection{CENÁRIO 3}

As matrizes de julgamento das alternativas propostas em relação a cada critério, bem como os valores da Razão de Consistência dos julgamentos para a situação característica do Cenário 3 são apresentados no Quadro 5. 
Quadro 5 - Matrizes de julgamento das alternativas em relação aos critérios, para o Cenário 3.

\begin{tabular}{|c|c|c|c|c|}
\hline CUSTO & $\bar{\xi}$ & $\bar{i}$ & 吾 & 产 \\
\hline API & 1 & $1 / 7$ & 3 & $1 / 5$ \\
\hline TPI & 7 & 1 & 7 & 2 \\
\hline TPI em linha & $1 / 3$ & $1 / 7$ & 1 & $1 / 8$ \\
\hline TPI com TE & 5 & $1 / 2$ & 8 & 1 \\
\hline \multicolumn{5}{|c|}{$\lambda_{\max }=4,157 ; \mathrm{IC}=0,052 ; \mathrm{RC}=0,058$} \\
\hline
\end{tabular}

\begin{tabular}{|c|c|c|c|c|}
\hline ESPAÇO & $\bar{z}$ & $\overline{\underline{G}}$ & 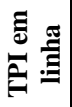 & 焉 \\
\hline API & 1 & $1 / 9$ & $1 / 4$ & $1 / 7$ \\
\hline TPI & 9 & 1 & 4 & 2 \\
\hline TPI em linha & 4 & $1 / 4$ & 1 & $1 / 5$ \\
\hline TPI com TE & 7 & $1 / 2$ & 5 & 1 \\
\hline \multicolumn{5}{|c|}{$\lambda_{\max }=4,160 ; \mathrm{IC}=0,053 ; \mathrm{RC}=0,059$} \\
\hline
\end{tabular}

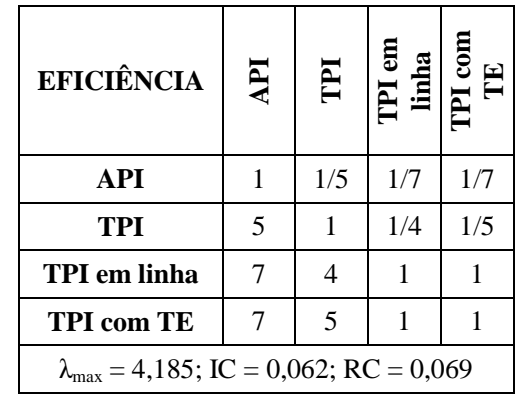

\begin{tabular}{|c|c|c|c|c|}
\hline $\begin{array}{c}\text { ATEND. } \\
\text { CONAMA } \\
\text { 357/430 }\end{array}$ & $\bar{z}$ & $\overline{\underline{A}}$ & 题 & 递 \\
\hline API & 1 & $1 / 3$ & $1 / 7$ & $1 / 9$ \\
\hline TPI & 3 & 1 & $1 / 5$ & $1 / 7$ \\
\hline TPI em linha & 7 & 5 & 1 & 1 \\
\hline TPI com TE & 9 & 7 & 1 & 1 \\
\hline \multicolumn{5}{|c|}{$\lambda_{\max }=4,096 ; \mathrm{IC}=0,032 ; \mathrm{RC}=0,035$} \\
\hline
\end{tabular}

\begin{tabular}{|c|c|c|c|c|}
\hline MANUTEN. & $\overline{\mathrm{z}}$ & $\overline{\underline{E}}$ & $\stackrel{g}{\Xi}$ & 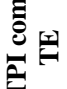 \\
\hline API & 1 & 3 & 5 & 4 \\
\hline TPI & $1 / 3$ & 1 & 3 & 1 \\
\hline TPI em linha & $1 / 5$ & $1 / 3$ & 1 & $1 / 6$ \\
\hline TPI com TE & $1 / 4$ & 1 & 6 & 1 \\
\hline \multicolumn{5}{|c|}{$\lambda_{\max }=4,224 ; \mathrm{IC}=0,075 ; \mathrm{RC}=0$} \\
\hline
\end{tabular}

O Quadro 6 apresenta a matriz de julgamento dos critérios avaliados em relação ao objetivo e a Razão de Consistência resultante deste julgamento, assim como os valores de Prioridade Média Local, Prioridade Global e o resultado final para o Cenário 3.

Quadro 6 - Matriz de julgamento dos critérios em relação ao objetivo, vetores Prioridade Média Local e Prioridade Global e resultado da seleção do SSAO, para o Cenário 3.

\begin{tabular}{|c|c|c|c|c|c|}
\hline $\begin{array}{c}\text { SELEÇÃO } \\
\text { DE SSAOO }\end{array}$ & $\begin{array}{l}0 \\
0 \\
0 \\
0\end{array}$ & 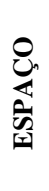 & 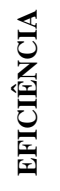 & 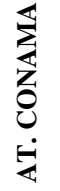 & 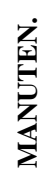 \\
\hline CUSTO & 1 & 2 & $1 / 3$ & $1 / 5$ & $1 / 2$ \\
\hline ESPAÇO & $1 / 2$ & 1 & $1 / 5$ & $1 / 3$ & $1 / 2$ \\
\hline EFICIÊNCIA & 3 & 5 & 1 & $1 / 4$ & 3 \\
\hline AT. CONAMA & 5 & 3 & 4 & 1 & 4 \\
\hline MANUTENÇÃO & 2 & 2 & $1 / 3$ & $1 / 4$ & 1 \\
\hline
\end{tabular}

\begin{tabular}{|c|c|c|c|c|c|c|}
\hline \multirow[b]{2}{*}{$\begin{array}{c}\text { SELEÇÃO } \\
\text { DE SSAO }\end{array}$} & \multicolumn{5}{|c|}{ VETORES PRIORIDADE - PML } & \multirow[b]{2}{*}{ 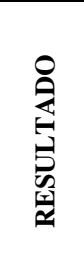 } \\
\hline & 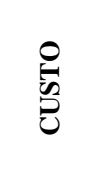 & 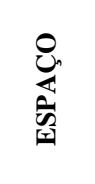 & 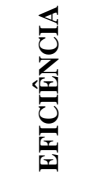 & 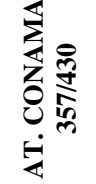 & 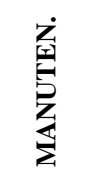 & \\
\hline API & 0,0933 & 0,0436 & 0,0476 & 0,0463 & 0,5263 & 0,1101 \\
\hline TPI & 0,5137 & 0,4886 & 0,1345 & 0,0934 & 0,1841 & 0,1826 \\
\hline TPI em linha & 0,0488 & 0,1205 & 0,3967 & 0,3989 & 0,0671 & 0,3048 \\
\hline TPI com TE & 0,3442 & 0,3472 & 0,4212 & 0,4614 & 0,2225 & 0,4025 \\
\hline $\begin{array}{l}\text { Prioridade } \\
\text { Global - PG }\end{array}$ & 0,0903 & 0,0748 & 0,2544 & 0,4567 & 0,124 & \\
\hline
\end{tabular}

Neste cenário, ao contrário dos cenários anteriores, o peso do critério custo não apresentou expressiva preferência em relação aos demais, representando $9 \%$ da prioridade para escolha do SSAO. Para esta situação, os maiores pesos foram obtidos para os critérios Eficiência e Atendimento da Resolução CONAMA 357/430, que representaram preferências de $25 \%$ e $46 \%$, respectivamente. O critério espaço foi o que apresentou a menor prioridade, $7 \%$, enquanto que o valor do peso do critério manutenção resultou em $12 \%$.

Os resultados da seleção de SSAO para os três cenários estudados são apresentados na Figura 2. A alternativa de implantação de Sistema de Separação Água/Óleo do tipo API foi que obteve maior percentual de preferência para as situações dos Cenários 1 e 2. Estes cenários caracterizam-se por apresentar tráfego das principais fontes poluentes (aeronaves e veículos de apoio), baixo e médio, respectivamente, apresentando potencial de contaminação de baixo risco ao meio ambiente. Além disso, representam as áreas mais extensas nos aeroportos, como pistas de pouso e decolagem e pistas de táxi, resultando em SSAO que ocupam grandes espaços a custos elevados. Desta forma, o critério custo, seguido do critério espaço, foram os que apresentaram maiores pesos no julgamento. Por outro lado, como o 
potencial de ocorrência de vazamentos de óleo, graxas e combustíveis é baixo, representando baixos riscos de contaminação nestes dois cenários, foi dada menos importância aos critérios mais técnicos, ou seja, Atendimento da Resolução CONAMA 357/430, Eficiência e Manutenção, resultando no sistema que apresenta o custo mais baixo dentre as alternativas estudadas.

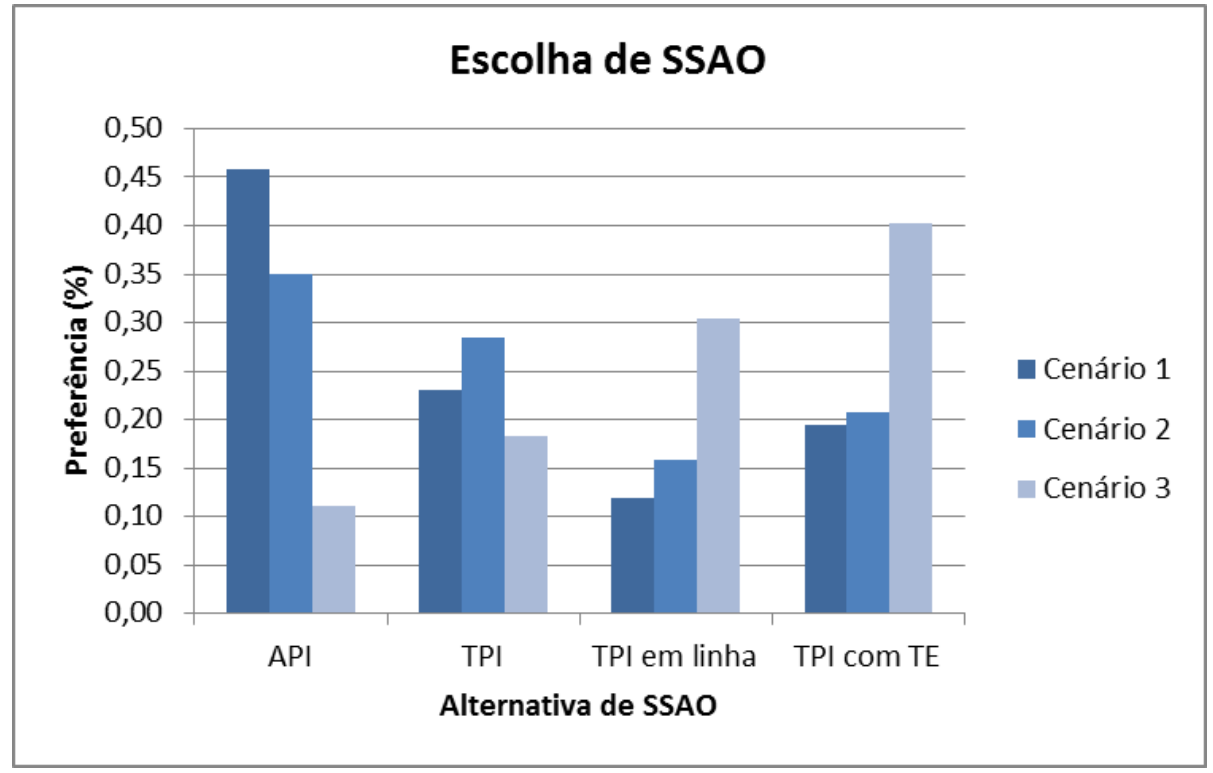

Figura 2 - Escolha de SSAO para diferentes cenários.

Com relação ao Cenário 3, característico de áreas com tráfego de aeronaves e veículos de apoio muito intenso e há permanência destas fontes poluentes por extensos períodos de tempo, a alternativa de SSAO do tipo TPI com TE, foi a que apresentou maior preferência. No julgamento pelo método AHP, ficou evidenciado que os critérios mais importantes para situações em que há elevado potencial de contaminação relacionam-se aos atributos técnicos do SSAO. Neste caso, o custo e a ocupação de espaço deixaram de ser os fatores principais, sendo preteridos pelos critérios, Atendimento da Resolução CONAMA 357/430, Eficiência e Manutenção, considerados mais adequados ao tratamento de efluentes contaminados com produtos oleosos resultantes da intensa movimentação de aeronaves e veículos de solo em pátios de estacionamento de aeronaves de aeroportos.

\section{CONCLUSÕES}

O método AHP foi estruturado com o objetivo de selecionar a melhor alternativa de implantação de Sistemas de Separação Água/Óleo em aeroportos, considerando diferentes cenários relacionados à movimentação de aeronaves e veículos de apoio ao solo, em três áreas aeroportuárias: pistas de pouso e decolagem; pistas de taxiamento e pátio de aeronaves.

A implantação de sistemas que utilizam separadores API foram os que se mostraram mais adequados para implantação nas áreas em que ocorrem os Cenários 1 e 2, onde os critérios de maior peso relacionam-se ao custo de implantação e à ocupação de espaço, em detrimento dos critérios técnicos que visam o atendimento à legislação ambiental, eficiência e manutenção do sistema.

Em situações de elevado volume de tráfego de aeronaves e veículos de apoio ao solo, que resultam em grande potencial para ocorrência de vazamentos de óleos e combustíveis, características do Cenário 3, a implantação de sistemas que utilizam separadores TPI com Tanques de Equalização, mostrou-se ser a mais adequada, com preferência aos atributos técnicos de Atendimento à Resolução CONAMA 357/430, Eficiência e Manutenção em relação ao Custo e Ocupação de Espaço. 
A aplicação do método AHP mostrou-se ser uma importante ferramenta para utilização pelos administradores de aeroportos no auxílio à tomada de decisão quanto da implantação de Sistemas de Separação Água/Óleos em aeroportos.

\section{REFERÊNCIAS}

[1] API, American Petroleum Institute. API Publication 421: Monographs on Refinery Environmental Control - Management of Water Discharges; Design and Operation of Oil/Water Separators. 1. ed. Washington D.C.: American Petroleum Institute, 1990.

[2] AZEVEDO, H. P. M. Descrição do Projeto do Sistema Separador de Água e Óleo do Aeroporto de Congonnhas. Trabalho de Conclusão de Curso (Graduação) - Universidade Anhembi Morumbi, São Paulo, 82p. 2007.

[3] BAStOS, A. L. A.; MATIAS, K. E.; DAMM, H.; LUNA, M. M. M. Modelo multicritério de apoio a decisão para seleção de fornecedores. VII Congresso Nacional de Excelência em Gestão, 2011. Disponível em: <http://www. excelenciaemgestao.org/Portals/2/documents/cneg7/anais/T11_0379_1703.pdf>. Acesso em: 17 set. 2014.

[4] CARVALHO, R. G.; KRUK, N. S. Critérios de dimensionamento para sistemas de separação água/óleo em aeroportos. In: XX Simpósio Brasileiro de Recursos Hídricos, Bento Gonçalves, nov. 2013.

[5] CARVAlho, R. G. Proposta de Dimensionamento de Sistema de Separação Água/Óleo em Aeroportos. Dissertação (Mestrado em Engenharia de Infraestrutura Aeronáutica) - Instituto Tecnológico de Aeronáutica, São José dos Campos/SP. 152p. 2014.

[6] FRANCO, L. A; MONTIBELLER, G. Problem structuring for multicriteria decision analysis interventions. Wiley Encyclopedia of Operations Research and Management Science. John Wiley and Sons, 2010.

[7] GOMES, L. F. A. M.; ARAYA, M. C. G.; CARIGNANO, C. Tomada de Decisões em Cenários Complexos: introdução aos métodos discretos do apoio multicritério à decisão. São Paulo: Thomson. 168p. 2004.

[8] SAATy, T. L. Método de Análise Hierárquica. São Paulo: McGraw-Hill, Makron, 1991.

[9] SAATY, T. L. Decision making for leaders. Pittsburg, USA: WS. Publications, 2000.

[10] SAATY, T. L. Decision making with analytic hierarchy process. International Journal Services Sciences, v.1, n.1, Pittsburg, USA. 2008.

[11] SANTOS, F. S.; VIAGI, A. F. Uso do método AHP (Analytic Hierarchy Process) para otimizar a cadeia de suprimentos durante o desenvolvimento integrado de produtos. Anais do XII Simpósio de Administração da Produção, Logística e Aplicações Internacionais. 2009.

[12] VAIDYA, O. S.; KUMAR, S. Analytic Hierarchy Process: An overview of applications. European Journal of Operational Research. n.169, p.1-29, 2006.

[13] VIEIRA, G. H. Análise e Comparação dos Métodos de Decisão Multicritério AHP Clássico e Multiplicativo. Trabalho de Conclusão de Curso (Graduação) Instituto Tecnológico de Aeronáutica, São José dos Campos/SP, 53p. 2006. 